\title{
The dynamic relationship between emotional and physical states: an observational study of personal health records
}

This article was published in the following Dove Press journal:

Neuropsychiatric Disease and Treatment

9 February 2017

Number of times this article has been viewed

\section{Ye-Seul Lee' \\ Won-Mo Jung' \\ Hyunchul Jang ${ }^{2}$ \\ Sanghyun Kim² \\ Sun-Yong Chung ${ }^{3}$ \\ Younbyoung Chae'}

'Acupuncture and Meridian Science Research Center, College of Korean Medicine, Kyung Hee University, Seoul, ${ }^{2}$ Mibyeong Research Center, Korean Institute of Oriental Medicine, Daejeon, ${ }^{3}$ Department of Neuropsychiatry, College of Korean Medicine, Kyung Hee University, Seoul, Republic of Korea
Correspondence: Younbyoung Chae Acupuncture and Meridian Science Research Center, College of Korean Medicine, Kyung Hee University, I Hoegi-dong, Dongdaemun-gu, Seoul |30-70|, Republic of Korea

$\mathrm{Tel}+82296 \mid 2208$

Fax +8229632175

Email ybchae@khu.ac.kr
Objectives: Recently, there has been increasing interest in preventing and managing diseases both inside and outside medical institutions, and these concerns have supported the development of the individual Personal Health Record (PHR). Thus, the current study created a mobile platform called "Mind Mirror" to evaluate psychological and physical conditions and investigated whether PHRs would be a useful tool for assessment of the dynamic relationship between the emotional and physical conditions of an individual.

Methods: Mind Mirror was used to collect 30 days of observational data about emotional valence and the physical states of pain and fatigue from 20 healthy participants, and these data were used to analyze the dynamic relationship between emotional and physical conditions. Additionally, based on the cross-correlations between these three parameters, a multilevel multivariate regression model (mixed linear model [MLM]) was implemented.

Results: The strongest cross-correlation between emotional and physical conditions was at lag 0 , which implies that emotion and body condition changed concurrently. In the MLM, emotional valence was negatively associated with fatigue $(\beta=-0.233, P<0.001)$, fatigue was positively associated with pain $(\beta=0.250, P<0.001)$, and pain was positively associated with fatigue $(\beta=0.398, P<0.001)$.

Conclusion: Our study showed that emotional valence and one's physical condition negatively influenced one another, while fatigue and pain positively affected each other. These findings suggest that the mind and body interact instantaneously, in addition to providing a possible solution for the recording and management of health using a PHR on a daily basis.

Keywords: emotion, fatigue, pain, personal health record (PHR), time-series analysis

\section{Introduction}

Mobile health, which is the use of mobile communication and computing technologies for medicine and public health, is rapidly expanding. ${ }^{1,2}$ A personal health record (PHR) is defined as a health record created using mobile computing technologies in which health information and personal health data are maintained by the patient. ${ }^{3}$ Models of PHR vary across a large range. One PHR model utilizes patient-generated data about health and lifestyle that are recorded using a personal computer or Web application and that help address specific health concerns. ${ }^{4,5}$ Because a PHR records health-related data generated by a patient, it is not only a repository of that patient's data but also a tool that facilitates interactions between the medical provider and patient via the provision of related health information. ${ }^{6-8}$ Additionally, because technologies are designed to streamline the diagnosis and treatment processes, and big data analytics offer novel perspectives regarding the contribution of health data to health care, PHRs 
provide the opportunity for a greater degree of interaction when managing health in one's daily life. ${ }^{9}$

Several recent studies have used PHRs to examine patient health and activity levels or to assess the management of patients through interventions outside medical institutions. Druss et $\mathrm{a}^{10}$ evaluated the effects of electronic PHRs on the quality of medical care in 170 patients with serious mental illnesses who were treated at a community medical center and found that the use of PHRs significantly improved the quality of medical care and increased the use of medical services by patients. Espie et al ${ }^{11}$ created a mobile health care application for the management of sleep disorders, which involved the development and psychometric validation of a brief scale (the Sleep Condition Indicator $[\mathrm{SCI}]$ ), to evaluate sleep disorders in everyday clinical practice. These authors found that web-based cognitive behavioral therapy had a positive effect on the treatment of insomnia. ${ }^{12}$ Park et al ${ }^{9}$ developed a PHR based on teen-specific needs to promote better self-awareness and chronic disease management and determined that chronic psychological or physical states require constant attention because the symptoms can fluctuate spontaneously over time. Moreover, although patients often focus on immediate problems, the use of PHRs may provide a tool that can gain the attention of individuals and remind them of these problems when not immediately apparent. ${ }^{9}$ Taken together, these findings suggest that PHRs constitute a useful tool to record the range of, and changes in, physical and psychological health states outside hospitals.

A growing body of research has called attention to the influence of emotion on health and the possible management of the relationship between these two variables. The instantiation of an affective state directly involves alterations in multiple physiological systems of the body, which leads to physiological responses that can directly influence physical health depending on the nature, frequency, and time course of the emotional state. ${ }^{13}$ Physiological responses are meant to be adaptive in the short term but can lead to maladaptive outcomes in the long term if not correctly regulated. ${ }^{14}$ Furthermore, recent evidence has sufficiently demonstrated the importance of comorbid relationships among emotional, psychological, and physical symptoms. ${ }^{15-20}$ For example, a worldwide survey using a national representative sample identified an association between chronic pain and mental disorders. ${ }^{21-23}$ Similarly, there is a growing consensus that negative emotions influence the development of cardiovascular disease ${ }^{24}$ and that chronic digestive disorders are closely linked with a variety of psychological disorders, including depression. ${ }^{25,26}$ Taken together, these studies provide a clear indication that chronic physical symptoms are best understood in the context of psychological factors. In this respect, the use of PHRs could provide a strategy for improving medical care for patients with comorbid psychological and physical illnesses.

In the current study, a mobile application called "Mind Mirror" was developed as a PHR to evaluate the daily affective states and physical conditions of individual patients. Using participant-generated health records, detailed information on health was collected on a daily basis through the participants' engagement and self-monitoring. This mobile platform was developed in order to collect health records while maximizing the patient's self-assessment in terms of paying attention to their physical condition and feelings throughout the day. A well-known example of self-assessment of daily experiences is the day reconstruction method (DRM), in which a participant reviews daily affective experiences and the subjective feelings. ${ }^{27,28}$ This was a 30-day observational study that aimed to characterize the dynamic relationship between the emotional state of patients and their physical states, including pain and fatigue, and to determine whether self-recorded tools such as PHRs would be useful for the assessment of the relationship between an individual's emotional and physical conditions.

\section{Methods \\ Participants}

All participants were recruited via an online advertisement posted on the Internet. The inclusion criteria for the current study were as follows: participants were required to 1) be between 20 and 40 years of age, 2) have no history of any neuropsychological disorder or acute or chronic pain disorder, and 3) not be taking any type of medication. Written informed consent was obtained prior to participation. This study was conducted in accordance with the guidelines issued by the human subjects committee and approved by the institutional review board of Kyung Hee University in Seoul, Republic of Korea.

\section{Study design and procedures}

The primary goals for the Mind Mirror mobile application were to assess the daily emotional and physical changes of patients and to provide a platform from which large-scale data could be collected and analyzed. A novel user interface format was applied to measure the affective and physical states of a participant every 2 hours from 9:00 AM to 9:00 PM for 30 days. The natural starting point of research on the dynamic nature of these symptoms lies in the analysis of symptoms measured over different time points. The analysis 
of time series data requires detailed records that reflect subtle changes in the emotional and physical conditions of individuals over time within a single day.

In the current study, participants completed the entries of their daily emotional and physical states using the Mind Mirror software; they were instructed to complete the data input into the application at night prior to going to bed. Data regarding emotional valence, fatigue, and pain were entered by the participants over 30 days at seven time points each day between 9:00 AM (09:00) and 9:00 PM (21:00). Of the eligible participants $(n=21)$, one participant was unable to finish the study due to loss of mobile phone; thus, the final data analyses in the current study included 20 participants (eight females, mean age $=24.7$ years) .

\section{Development of software for Mind Mirror}

For the current study, an iPhone Operating System (iOS) software application was developed using Xcode 6.4 and the programming language "Swift"; this application required iOS 8.4 Software Development Kit (SDK) or higher. Explicit methods were used to record the subjective ratings of the participants regarding their physical conditions and subjective valences of daily emotion. The explicit method collects data on emotions and physical conditions that the participant cognitively feels by asking direct questions or presenting straightforward tasks regarding their daily condition. ${ }^{29}$ Upon turning on the application, a title page with a logo, as well as a message that reads "Start new record", appears. Once the participant has clicked on this message, the application begins its initial steps. The participants entered this application once a day (Figure 1A).

\section{Measurements of emotional and physical states using PHRs \\ Measurement of emotional states}

This application applied two main methods to measure explicit emotions or feelings that were presented as tasks for the participants to complete on a daily basis. The first measure was designed to quantitatively visualize positive or negative affective states. On the screen, three auxiliary lines with the numbers 5,0 , and -5 next to each line were provided. The words "Pleasant" and "Unpleasant" were placed beside the numbers 5 and -5 to indicate positive and negative emotional valences, respectively. Circles that could be dragged were located on the middle line, and time points shown in units of 2 hours from 9:00 AM to 9:00 PM were visible. The participants moved the circles up and down on a vertical plane within the range of -5 to 5 to express their emotional states (Figure 1B).
The second measure depicted six basic emotions as described by Ekman: anger, disgust, fear, joy, sadness, and surprise. ${ }^{13}$ The emotions were presented on each vertex of a hexagon, and the relative intensities of the six distinct emotions were defined as the circumplex model. Moreover, 10 small circles, which were depicted as drops of water, were presented at the center of the hexagon, and a single drop of water moved to attach to the corresponding emotion when the user touched any of the six emotions a single time. Each participant was asked to touch the emotions in order to distribute the 10 drops of water as a description of their overall emotional state for the day; it was not necessary to use all 10 drops of water (Figure 1C). The data from this measure were collected from the participants as well, but not used for the study analysis.

\section{Measurement of physical states}

The first step in determining a participant's physical state involved asking questions about the weather during each day using simple pictures. After choosing the weather, the participants were asked to complete their assessment of the length and quality of the previous night's sleep and to evaluate their digestive function throughout the day. The last step involved assessing one chronic condition that the participant wanted to monitor for 30 days, which was reported prior to starting the study; the personalized chronic symptoms ranged from eye irritation to dizziness. To assess the chronic symptom, each participant moved a button across a bar that was labeled at each end to indicate the "Worst" and "Best" conditions. This system applied a variation of the visual analog scale (VAS) in which performances in computer-based and Web-based research have been validated. ${ }^{30}$ While categorical scales reach an ordinal-scale level, the VAS extends the precision and discrimination of daily reports (Figure 1D). The data from this measure were collected from the healthy participants and not used for the study analysis.

Two main methods were applied to measure physical conditions according to time. Like the emotional tasks, these were presented as tasks for the participants to complete on a daily basis and quantitatively described the positive or negative physical states of each participant. The first task involved assessing one's overall pain and the second task involved assessing one's overall fatigue throughout the day. On the screen, three auxiliary lines with the numbers 10 and zero next to the top and bottom lines, respectively, were provided. The words "Extremely severe" and "None" were placed besides the numbers 10 and zero to indicate the intensities of pain and fatigue. Circles that could be dragged were located on the middle line and time points shown in 
A

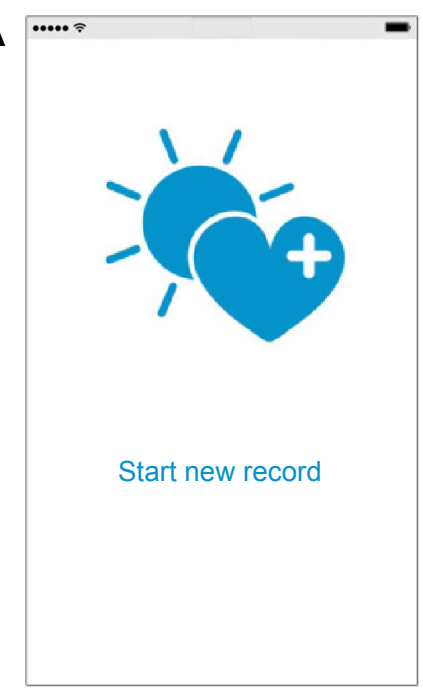

D

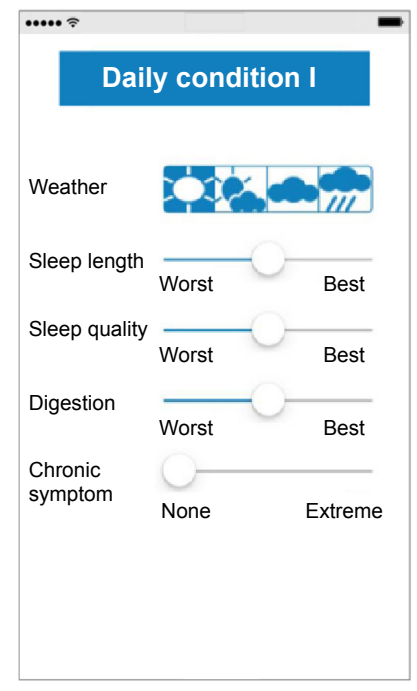

B

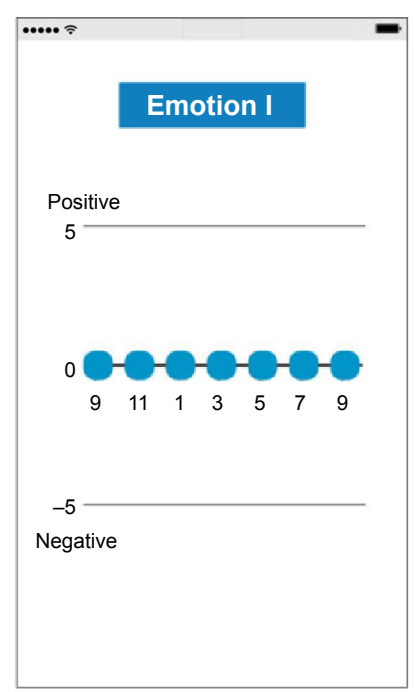

E

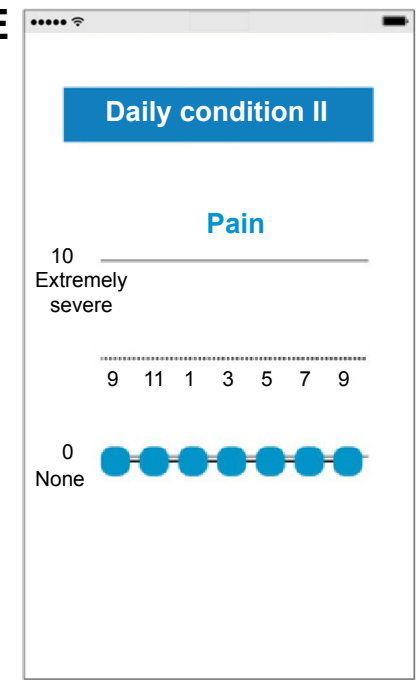

C

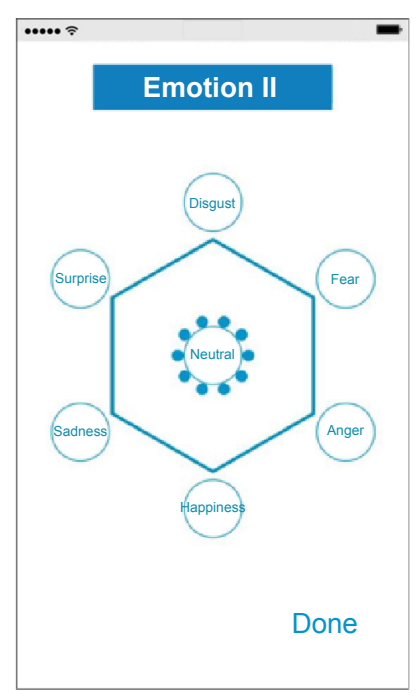

$\mathbf{F}$

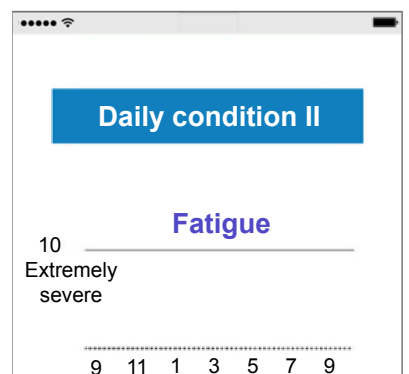

0

None

Figure I Structure of the "Mind Mirror" mobile application.

Notes: (A) Starting page for the Mind Mirror mobile application; clicking on "Start new record" changes the application screen to the next figure. (B) Explicit measurement of Emotion I. The blue circle in the middle line is moved by the participant to express the level of emotional valence, which has a range from -5 to 5 and is measured every 2 hours from 9:00 AM to 9:00 PM. (C) Explicit measurement of Emotion II. The hexagon represents a circumplex model of six distinct emotions according to Ekman: happiness, anger, fear, disgust, surprise, and sadness. A single click on one of the six emotions moves the blue circle at the center to the clicked emotion. The participant can divide 10 blue circles into different emotions to illustrate the type and strength of the emotions that he or she felt during the day. (D) Basic health information of a patient throughout the day. The participant was asked to choose the weather of the day by clicking on one of the various weather icons. They were then asked to note the length and quality of sleep the previous night by moving a button within a bar that ranged from "Worst" to "Best". Similarly, the participants rated digestive function throughout the day. Chronic symptoms were identified prior to the trial; moving the button within a bar that ranged from "None" to "Extremely severe" changed the severity of the daily symptoms over 30 days, which the patient could track. (E) Measurement of pain. The blue circle in the bottom line was moved by the participant to express the level of pain during the day. (F) Measurement of fatigue. The blue circle in the middle line was moved by the participant to express the level of fatigue during the day.

units of 2 hours from 9:00 AM to 9:00 PM were visible. The participants moved the circles up and down on a vertical plane within the range of $10-0$ to express their physical states throughout the day; the same procedure was applied for both pain and fatigue (Figure 1E and F).

\section{Data processing and analysis}

Because this study focused on the dynamic relationship between emotional and physical states, records with a more frequent temporal resolution (units of 2 hours) were analyzed; thus, a time series analysis of the data of 20 participants was conducted to determine the correlations between the emotional and physical states. The collected data of the participants during the 30-day study were placed in a property list (.plist) file format that was converted to a comma separated values (.csv) file for the data analyses. There were three different categories of time series data - emotional valence, pain, and fatigue which illustrated the experiences and emotional states of the participants on a daily basis. The 30-day data from 20 participants had average missing assessments of 1.7 days. 
An interpolation process was applied to complete the data for time points between 11:00 PM and 7:00 AM when the participants were assumed to be asleep. Using this preprocessing procedure, five time points for a single night, or 150 time points in total, were filled in to complete the data for the 30-day study period. To fill in the empty time points, the function "na.interp" in the "forecast" package of R (http:// www.R-project.org), which assumes a linear relationship, was used to complete the data set.

Following the preprocessing procedure, three sets of time series data for each of the 20 participants were available for data analysis. A logarithmic transformation was applied to each of the emotional, pain, and fatigue series to ensure the normality and homogeneity of variance of the residuals. Cross-correlations between the emotional valence and pain, emotional valence and fatigue, as well as fatigue and pain time series were analyzed by participant, and the correlation coefficients were averaged to visualize the correlation analysis. R software (version 3.2.3, "Wooden Christmas Tree") and R Studio (version 0.99.892) were used for the data analyses.

\section{Mixed linear model (MLM)}

To investigate the possible relationships between emotional valence and physical states using the data of the 20 participants, a regression model was applied with consideration given for the random effects of the participants. When selecting a regression model to determine whether there were associations among emotion, fatigue, and pain, it was assumed that there were concurrent dynamics between emotion and the body based on cross-correlation results. Accordingly, a mixed model for multilevel data with a combination of between-subject and within-subject factors was used.

While some methods for time series analyses account for random effects in multilevel data, ${ }^{31}$ many other methods examine the concurrent changes between multiple time series without lagged effects. Thus, a single model was selected to analyze the three series to determine whether any associations existed. The multilevel regression model, or the MLM, ${ }^{32,33}$ allows for the estimation of hierarchically structured longitudinal data on the individual and group levels. For the current data, a mixed model with participants as the random effect without temporal dislocation was applied because cross-correlation analyses of the series unanimously showed the highest correlation at lag 0 . Based on the regression beta coefficients, the dynamic structure between emotional valence, pain, and fatigue was visualized in a network; the green line indicates a positive relationship, whereas the red line indicates a negative relationship.

\section{Results \\ Correlations between emotional and physical states}

Following the data preprocessing, an augmented DickeyFuller test analyzing the three time series with no lagged differences indicated that the logarithmic emotion series, pain series, and fatigue series were each stationary time series. Thus, no temporal dislocation was required to meet the stationarity requirement for the regression analysis.

The correlation analysis revealed that there were negative correlations between emotional valence and fatigue, as well as emotional valence and pain, while pain and fatigue were positively correlated; all three relationships showed the highest correlations at lag 0 . Figure 2 depicts the plots of the mean cross-correlation values among the participants between the emotional valence and fatigue, emotional valence and pain, and pain and fatigue series. The crosscorrelation was the strongest at lag 0 ; at lag 0 , the mean of the participants' cross-correlation coefficient (ccf) values
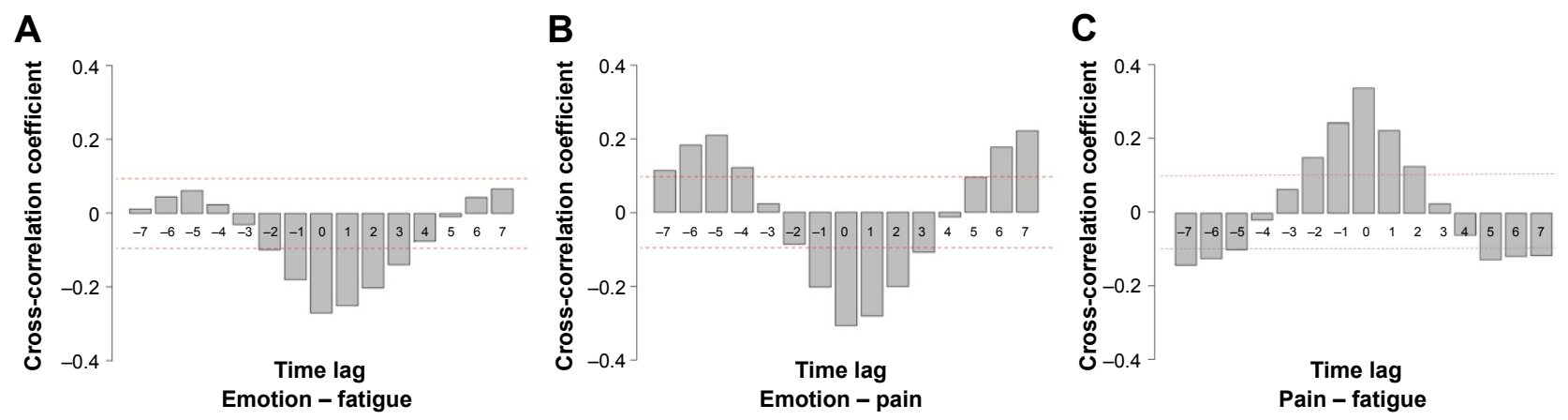

Figure 2 Group-level correlogram of correlation coefficients according to the time lags among emotion, fatigue, and pain.

Notes: Cross-correlation analyses of the time series of emotion, fatigue, and pain conducted by participant; the 20 acquired correlation coefficients in each set (emotion and fatigue, emotion and pain, as well as pain and fatigue) were averaged among the 20 participants and shown in the correlogram. The threshold was \pm 0.96 , which is the threshold level for cross-correlations with 15 time points. (A) Group-level cross-correlation analysis of emotion and fatigue by time. (B) Group-level cross-correlation analysis of emotion and pain by time. (C) Group-level cross-correlation analysis of pain and fatigue by time. 
Table I Mixed generalized linear regression model of the multiple time series for emotional valence, fatigue, and pain

\begin{tabular}{|c|c|c|c|c|c|c|c|c|c|}
\hline & \multicolumn{3}{|l|}{ Model I } & \multicolumn{3}{|l|}{ Model 2} & \multicolumn{3}{|l|}{ Model 3} \\
\hline & \multicolumn{3}{|c|}{ (Pain $\leftarrow$ Emotion + Fatigue $)$} & \multicolumn{3}{|c|}{ (Fatigue $\leftarrow$ Emotion + Pain) } & \multicolumn{3}{|c|}{ (Emotion $\leftarrow$ Pain + Fatigue) } \\
\hline & $\beta$ & SE & $P$-value & $\beta$ & SE & $P$-value & $\beta$ & SE & $P$-value \\
\hline Emotion & -0.021 & 0.011 & 0.063 & -0.233 & 0.014 & $<0.001$ & - & - & - \\
\hline Fatigue & 0.250 & 0.009 & $<0.001$ & - & - & - & -0.150 & 0.009 & $<0.001$ \\
\hline Pain & - & - & - & 0.398 & 0.014 & $<0.001$ & -0.022 & 0.012 & 0.063 \\
\hline Log-likelihood & $-|5,408|$. & & & $-15,536.2$ & & & $-17,162.2$ & & \\
\hline $\mathrm{AIC}$ & $30,830.2$ & & & $31,086.3$ & & & $34,338.5$ & & \\
\hline $\mathrm{BIC}$ & $30,878.7$ & & & $31,134.8$ & & & $34,387.0$ & & \\
\hline
\end{tabular}

Abbreviations: AIC, Akaike information criterion; BIC, Bayesian information criterion; SE, standard error.

were as follows: Emotion-Fatigue $=-0.289(P<0.001)$; Emotion-Pain $=-0.208(P=0.007)$; and Pain-Fatigue $=0.321$, $(P<0.001)$.

\section{Multilevel regression model of the emotional and physical states}

There was no heterogeneity in the random slopes of the between-subjects factor. Table 1 summarizes the dynamic interactions among the three series $(\beta)$, as well as the Akaike information criterion (AIC), Bayesian information criterion (BIC), and log likelihood of each model. The MLM revealed that fatigue was negatively associated with emotional valence $(\beta=-0.233, P<0.001)$ and positively associated with pain ( $\beta=0.398, P<0.001)$. Additionally, pain was significantly associated with fatigue $(\beta=0.250, P<0.001)$ and emotional valence was significantly more negative as fatigue increased ( $\beta=-0.150, P<0.001)$. Emotional valence also exhibited a negative change as pain increased $(\beta=-0.022, P=0.063)$ and pain increased as emotion changed negatively $(\beta=-0.021$, $P=0.063$ ), but these results were not statistically significant. The dynamic network structure between emotional valence, pain, and fatigue is presented in Figure 3.

\section{Discussion}

The current study developed a PHR called Mind Mirror for the recording of daily emotions, pain, and fatigue. By using explicit methods to retrospectively record changes in emotional valence, pain, and fatigue every 2 hours on a daily basis, this mobile platform enabled the collection of a set of individualized time series data that illustrated emotional and physical changes in individual patients. The analyses revealed that there were dynamic relationships between daily emotional and physical states in healthy participants. At lag 0 , emotion and fatigue were negatively correlated, while fatigue and pain were positively correlated. In the dynamic structural network produced by the MLM, the body, or the physical states of pain and fatigue, instantly interacted with emotional valence. Additionally, the overall physical states of fatigue and pain seemed to positively interact with each other, which may have interactively facilitated either the improvement or worsening of a condition. The DRM is one way of self-assessment on the daily affective experiences..$^{27,28}$ While self-assessments of the recent affective experiences such as DRM have been studied to contribute to subjective well-being, our study focused on not only the emotional states but also the participant's physical symptoms that may affect their daily experiences. By doing so, this mobile platform aimed to provide a tool for assessing daily situations, as well as a daily record of psychological and physical states.

The current findings suggest that emotional valence and fatigue directly influence one another and that emotional valence and pain influence one another through the

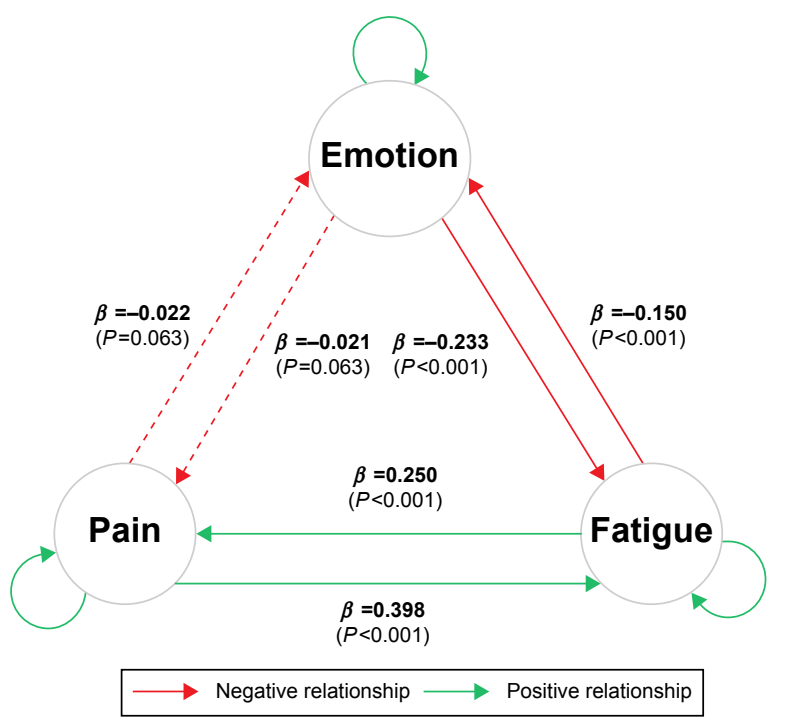

Figure 3 Dynamic network among emotional valence, fatigue, and pain according to a multilevel regression analysis.

Notes: The green arrow indicates a positive relationship, and the red arrow indicates a negative relationship. A solid line indicates a significant association, and a dotted line indicates a nonsignificant relationship. 
mediating symptom of fatigue, even in healthy individuals. These findings agree with those of previous studies that identified a relationship between negative emotions and physical states. ${ }^{20-24,35-38}$ For example, there are strong genetic links between chronic widespread musculoskeletal pain and fatigue as well as between chronic widespread pain and depression. ${ }^{34}$ Other studies have identified a close relationship between emotional valence and one's physical condition. Accordingly, chronic pain is associated with mental disorders, and negative emotions are known to influence the development of cardiovascular diseases. ${ }^{21,22,24,35}$ Pain disorders without a definite cause, including fibromyalgia (in which the most common symptoms are pain, fatigue, and depression), affect patients in such a way that treatments help to relieve symptoms but not to eliminate the cause of the disorders. ${ }^{34,36}$ Diseases such as cancer are associated with a variety of emotional symptoms, including anxiety and depression, as well as physical symptoms, including pain and fatigue..$^{20,37}$ On the other hand, although there is evidence that mood influences pain disorders, the influence of a negative emotional state on one's physical state has also been shown to be either selective, general, or unclear. ${ }^{23,38} \mathrm{~A}$ 30-day observational study demonstrated that emotions affect healthy individuals and that this influence is instantly evident even in the absence of awareness of a patient. ${ }^{24}$

In this respect, PHRs may provide a possible strategy for improving medical care for patients with comorbid psychological and physical illnesses. ${ }^{39}$ Thus, the findings of the current study can be applied to health management by medical professionals and institutions for the purpose of analyzing daily emotions that can lead to diseases as well as for predicting possible changes in daily emotions. Participant-generated health data, such as those produced by the current study, hold potential for the self-monitoring and daily measurement of health conditions, which will aid in the investigation of possible relationships between an individual's emotions and chronic physical symptoms outside the hospital. In a more general sense, PHRs point to the promise of health technologies for managing health and preventing the occurrence or worsening of various disorders among members of the general population who have access to mobile technology. Moreover, a variety of diverse information can be obtained using this format, depending on the target population, including inpatients within medical facilities, outpatients who make regular visits, and healthy individuals who are yet to receive medical checkups. Reduced gaps in health-related information may aid in the diagnoses of individuals who do not have a clear cause for their symptoms.
As these technologies are developed and distributed in the near future, it will be essential to ensure that they are available for and tested in patients with psychological and/or psychosomatic symptoms.

The current study has several limitations that must be noted. This study found marked and direct, but not significant, relationships between pain and emotion, which may be interpreted from two different perspectives. First, this study was conducted with a limited number of participants and, thus, further investigations are needed to compare these findings with those of other studies in order to establish generalizability with other age groups and health settings with a larger population. Second, this study was conducted using only a subset of participants with regular emotional and physical health statuses and, thus, they may have had different relationships between their emotional and physical states relative to chronic disease patients. For instance, mental disorders, including depression, are known to be comorbid with chronic pain. ${ }^{40}$ Thus, further research is needed to examine the benefits of this application and other types of novel technologies in different patient groups. Furthermore, the mobile application-based PHR used in the current study required that the participants enter all data and access their records using smartphones, which may have limited the population eligible for this intervention. This data collection procedure has also limited data quality control. Third, the retrospective assessment of the pain, fatigue, and emotional valence by the participants, as indicated in our study, may introduce recall bias. While the strategy to use this mobile platform minimized any missing values during the trial, the recall bias may have consequences for the data quality. One way of avoiding this problem, as used by other mobile platforms, is to prompt the participants several times during the day. Finally, the current analyses did not include data collected from the circumplex model of categorical emotion or other items, including sleep or other chronic symptoms, because it was focused on the temporal dynamics of the relationships between the emotional and physical states of patients. It would be interesting to investigate the interplay between different types of emotions and physical symptoms in the future. Future studies in which a large sample size and the analysis of a combination of other items are utilized when an application-based PHR is widely used worldwide should be conducted.

\section{Conclusion}

The current 30-day observational study examined relationships among pain, fatigue, and emotional valence and 
provided evidence that the physical condition and emotional state of healthy participants are interrelated. Positive changes in emotional valence were associated with improvements in physical condition via decreases in pain and fatigue, while negative changes in emotional valence were associated with the aggravation of pain and fatigue. By measuring differences in daily emotions, the current study provided basic information about both emotional and physical health in daily life using a mobile platform that recorded emotional and physical changes throughout the day on a daily basis. These findings also suggest that further data collection and analyses will contribute to the ability to predict an individual's emotional and physical health conditions, which would aid in the management of an individual's health on a daily basis.

\section{Acknowledgments}

This research was supported by a grant-in-aid from the Korea Institute of Oriental Medicine (grant number K15511). The funders had no role in study design, data collection and analysis, decision to publish, or preparation of the manuscript.

\section{Disclosure}

The authors report no conflicts of interest in this work.

\section{References}

1. Free C, Phillips G, Watson L, et al. The effectiveness of mobile-health technologies to improve health care service delivery processes: a systematic review and meta-analysis. PLoS Med. 2013;10(1):e1001363.

2. Akter S, D'Ambra J, Ray P. Development and validation of an instrument to measure user perceived service quality of mHealth. Inf Manag. 2013;50(4):181-195.

3. Tang PC, Ash JS, Bates DW, Overhage JM, Sands DZ. Personal health records: definitions, benefits, and strategies for overcoming barriers to adoption. J Am Med Inf Assoc. 2006;13(2):121-126.

4. Huba N, Zhang Y. Designing patient-centered personal health records (PHRs): health care professionals' perspective on patient-generated data. J Med Syst. 2012;36(6):3893-3905.

5. Pagliari C, Detmer D, Singleton P. Potential of electronic personal health records. BMJ. 2007;335(7615):330-333.

6. Akter S, D'Ambra J, Ray P. Trustworthiness in mHealth information services: an assessment of a hierarchical model with mediating and moderating effects using partial least squares (PLS). J Am Soc Inform Sci Technol. 2011;62(1):100-116.

7. Ford EW, Hesse BW, Huerta TR. Personal health record use in the United States: forecasting future adoption levels. J Med Internet Res. 2016; 18(3):e73.

8. Patel MS, Asch DA, Volpp KG. Wearable devices as facilitators, not drivers, of health behavior change. JAMA. 2015;313(5):459-460.

9. Park T, Chira P, Miller K, Nugent L. Living profiles: an example of user-centered design in developing a teen-oriented personal health record. Pers Ubiquit Comput. 2014;19:69-77.

10. Druss BG, Ji X, Glick G, von Esenwein SA. Randomized trial of an electronic personal health record for patients with serious mental illnesses. Am J Psychiatry. 2014;171(3):360-368.

11. Espie CA, Kyle SD, Hames P, Gardani M, Fleming L, Cape J. The Sleep Condition Indicator: a clinical screening tool to evaluate insomnia disorder. BMJ Open. 2014;4(3):e004183.
12. Espie CA, Kyle SD, Miller CB, Ong J, Hames P, Fleming L. Attribution, cognition and psychopathology in persistent insomnia disorder: outcome and mediation analysis from a randomized placebo-controlled trial of online cognitive behavioural therapy. Sleep Med. 2014;15(8): 913-917.

13. DeSteno D, Gross JJ, Kubzansky L. Affective science and health: the importance of emotion and emotion regulation. Health Psychol. 2013;32(5):474-486.

14. Extremera N, Rey L. The moderator role of emotion regulation ability in the link between stress and well-being. Front Psychol. 2015;6:1632.

15. Cohen S, Rodriquez MS. Pathways linking affective disturbances and physical disorders. Health Psychol. 1995;14(5):374-380.

16. Benjamin S, Morris S, McBeth J, Macfarlane GJ, Silman AJ. The association between chronic widespread pain and mental disorder: a population-based study. Arthritis Rheum. 2000;43(3):561-567.

17. Mittermaier C, Dejaco C, Waldhoer T, et al. Impact of depressive mood on relapse in patients with inflammatory bowel disease: a prospective 18-month follow-up study. Psychosom Med. 2004;66(1):79-84.

18. Tunks ER, Crook J, Weir R. Epidemiology of chronic pain with psychological comorbidity: prevalence, risk, course, and prognosis. Can J Psychiatry. 2008;53(4):224-234.

19. Gerontoukou EI, Michaelidoy S, Rekleiti M, Saridi M, Souliotis K. Investigation of anxiety and depression in patients with chronic diseases. Health Psychol Res. 2015;3(2):2123.

20. Badr H, Basen-Engquist K, Carmack Taylor CL, de Moor C. Mood states associated with transitory physical symptoms among breast and ovarian cancer survivors. J Behav Med. 2006;29(5):461-475.

21. Demyttenaere K, Bruffaerts R, Lee S, et al. Mental disorders among persons with chronic back or neck pain: results from the World Mental Health Surveys. Pain. 2007;129(3):332-342.

22. Gureje O, Von Korff M, Kola L, et al. The relation between multiple pains and mental disorders: results from the World Mental Health Surveys. Pain. 2008;135(1-2):82-91.

23. McWilliams LA, Cox BJ, Enns MW. Mood and anxiety disorders associated with chronic pain: an examination in a nationally representative sample. Pain. 2003;106(1-2):127-133.

24. Roest AM, Martens EJ, de Jonge P, Denollet J. Anxiety and risk of incident coronary heart disease: a meta-analysis. J Am Coll Cardiol. 2010;56(1):38-46.

25. Locke GR 3rd, Weaver AL, Melton LJ 3rd, Talley NJ. Psychosocial factors are linked to functional gastrointestinal disorders: a population based nested case-control study. Am J Gastroenterol. 2004;99(2):350-357.

26. Hartono JL, Mahadeva S, Goh KL. Anxiety and depression in various functional gastrointestinal disorders: do differences exist? J Dig Dis. 2012;13(5):252-257.

27. Kahneman D, Krueger AB, Schkade DA, Schwarz N, Stone AA. A survey method for characterizing daily life experience: the day reconstruction method. Science. 2004;306(5702):1776-1780.

28. Miret M, Caballero FF, Mathur A, et al. Validation of a measure of subjective well-being: an abbreviated version of the day reconstruction method. PLoS One. 2012;7(8):e43887.

29. Lane RD, Nadel L. Cognitive Neuroscience of Emotion. USA: Oxford University Press; 2002.

30. Reips UD, Funke F. Interval-level measurement with visual analogue scales in Internet-based research: VAS generator. Behav Res Methods. 2008;40(3):699-704.

31. Bringmann LF, Vissers N, Wichers M, et al. A network approach to psychopathology: new insights into clinical longitudinal data. PLoS One. 2013;8(4):e60188.

32. Fanavoll R, Nilsen TI, Holtermann A, Mork PJ. Psychosocial work stress, leisure time physical exercise and the risk of chronic pain in the neck/shoulders: longitudinal data from the Norwegian HUNT Study. Int J Occup Med Environ Health. 2016;29(4):585-595.

33. Rodríguez G. Multilevel generalized linear models. In: de Leeuw J, Meijer E, editors. Handbook of Multilevel Analysis. London: Springer; 2008:335-376. 
34. Burri A, Ogata S, Livshits G, Williams F. The association between chronic widespread musculoskeletal pain, depression and fatigue is genetically mediated. PLoS One. 2015;10(11):e0140289.

35. Lane RD, Carmichael C, Reis HT. Differentiation in the momentary rating of somatic symptoms covaries with trait emotional awareness in patients at risk for sudden cardiac death. Psychosom Med. 2011;73(2): 185-192.

36. Bar-On Kalfon T, Gal G, Shorer R, Ablin JN. Cognitive functioning in fibromyalgia: the central role of effort. J Psychosom Res. 2016;87: 30-36.

37. Fleming L, Randell K, Harvey CJ, Espie CA. Does cognitive behaviour therapy for insomnia reduce clinical levels of fatigue, anxiety and depression in cancer patients? Psychooncology. 2014;23(6):679-684.
38. Marangell LB, Clauw DJ, Choy E, et al. Comparative pain and mood effects in patients with comorbid fibromyalgia and major depressive disorder: secondary analyses of four pooled randomized controlled trials of duloxetine. Pain. 2011;152(1):31-37.

39. Estrin D, Sim I. Health care delivery. Open mHealth architecture: an engine for health care innovation. Science. 2010;330(6005):759-760.

40. Jain R. The implications of pain and physical symptoms in depression. J Clin Psychiatry. 2009;70(6):e19.

\section{Publish your work in this journal}

Neuropsychiatric Disease and Treatment is an international, peerreviewed journal of clinical therapeutics and pharmacology focusing on concise rapid reporting of clinical or pre-clinical studies on a range of neuropsychiatric and neurological disorders. This journal is indexed on PubMed Central, the 'PsycINFO' database and CAS, and is the official journal of The International Neuropsychiatric Association (INA). The manuscript management system is completely online and includes a very quick and fair peer-review system, which is all easy to use. Visit http://www.dovepress.com/testimonials.php to read real quotes from published authors.

Submit your manuscript here: http://www.dovepress.com/neuropsychiatric-disease-and-treatment-journal 